\title{
Radiocarbon
}

1975

\section{BIRMINGHAM UNIVERSITY RADIOCARBON DATES IX}

F W SHOTTON, R E G WILLIAMS and A S JOHNSON

Department of Geological Sciences, University of Birmingham, Birmingham, England

The following list of dates contains the majority of measurements made during 1974, $i e$, since our last list (R, 1974, v 16, p 285-303).

Age calculations are based on $95 \%$ activity of the NBS oxalic acid standard computed from the Libby half-life of $5570 \pm 30$ yr. Errors quoted refer only to the standard deviation $(\mathbf{1} \boldsymbol{\sigma})$ calculated from a statistical analysis of sample, background, and standard count rates.

${ }^{13} \mathrm{C} /{ }^{12} \mathrm{C}$ ratios are measured directly on all methane gas samples as previously described (R, 1973, v 15, p 451) and ages are corrected for $\delta^{1:} \mathrm{C}$ deviations.

Sample preparation and pretreatment continue as before (R, 1969, $\mathrm{v} 11, \mathrm{p} 263)$. Where sample size was insufficient for full pretreatment, details of procedure accompany the result. For bone samples, we use the extraction method of Longin (1971) based on the solubility of collagen in slightly acidic hot water.

\section{ACKNOWLEDGMENTS}

We particularly wish to thank Lina Salvini for sample preparation and pretreatment. Sample descriptions are based on information provided by submitters and collectors.

\section{SAMPLE DESCRIPTIONS}

I. GEOLOGIC SAMPLES

A. British Isles

\section{Cnoc Sligeach series, Isle of Oronsay, Scotland}

Shells from storm-beach gravel and midden material $30 \mathrm{~m} \mathrm{~S}$ of Cnoc Sligeach, I of Oronsay, Inner Hebrides, Argyllshire, Scotland $\left(56^{\circ} 01^{\prime} \mathrm{N}\right.$, $06^{\circ} 12^{\prime}$ W, Grid Ref NR 373889). Coll Aug 1973 and subm by W G Jardine, Dept Geol, Univ Glasgow.

\section{Birm-462.}

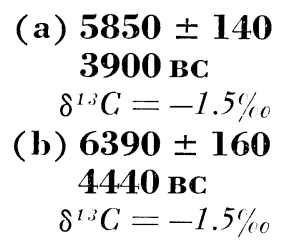

Valves of Patella from hard-packed layer of cobbles and limpets at $+10.97 \mathrm{~m}$ alt assoc with storm-beach formation. 
Birm-463.

(a) $8220 \pm 170$ $6270 \mathrm{BC}$

$\delta^{13} C=-0.1 \%$

(b) $7210 \pm 130$ 5260 BC $\delta^{13} \mathrm{C}=-1.2 \%$

Valves of Ostrea, Patella, Arctica, and Littorina (?) from layer of cobbles and limpets with charcoal at $+11.17 \mathrm{~m}$ alt assoc with periods of occasional occupation at times of storms.

\section{Birm-464.}

Single valve of Arctica islandica from layer of pebbles, charcoal, bone, and large shells at $+11.47 \mathrm{~m}$ alt, assoc with periods of occasional occupation at times of storm.

\section{Birm-465.}

(a) $6010 \pm 150$ $4060 \mathrm{BC}$ $\delta^{13} \mathrm{C}=-1.3 \%$

(b) $5900 \pm 150$ 3950 BC $\delta^{13} \mathrm{C}=-2.2 \%$ o

Valves of Pecten from layer ca $0.5 \mathrm{~m}$ deep, $+11.97 \mathrm{~m}$ alt, assoc with more permanent occupation period.

General Comment: outer fraction discarded, inner (a) and middle (b) fraction dated. Samples from pit within few meters from trench described by Bishop (1914) both with similar stratigraphy. Relationship between beach formation and site occupation of similar shell mound at Caistealnan-Gillean discussed in Birm-414 (R, 1974, v 16, p 290-291). Birm-462 appears anomalously young.

\section{Birm-481. Lawrence Edge Landslip, Derbyshire}

$$
\begin{gathered}
1970 \pm 100 \\
20 \mathrm{BC} \\
\delta^{13} C=-28.0 \%
\end{gathered}
$$

Wood (Betula), id by D Shimwell, ca $2.5 \mathrm{~m}$ deep at base of peat layer $2 \mathrm{~m}$ thick in iron-enriched deposit which passes into a true gley horizon, part of small earthflow derived from slump area of extensive landslip $\mathrm{N}$ of Lawrence Edge, Derbyshire $\left(53^{\circ} 29^{\prime} \mathrm{N}, 01^{\circ} 52^{\prime} \mathrm{W}\right.$, Grid Ref SK 085995). Coll Nov 1973 and subm by R H Johnson, Dept Geog, Univ Manchester. Comment (RHJ): dates termination of major earth movement in locality. 


\section{Birm-482. Sheldon, Warwickshire}

$11,970 \pm 340$ 10,020 вС

$\delta^{13} \mathrm{C}=-26.6 \%$

Plant material from 1.83 to $1.88 \mathrm{~m}$ deep in sandy peat filling hollow in glacial sand near Stirrup Cup Inn, Sheldon, Warwickshire $\left(52^{\circ} 27^{\prime} 50^{\prime \prime}\right.$ N, $01^{\circ} 46^{\prime} 15^{\prime \prime} \mathrm{W}$, Grid Ref SP 155853). Coll 1966 by C H S Sands; subm by $\mathrm{T}$ A Brown, Dept Geol, Univ Birmingham. Comment: $5 \mathrm{~cm}$ peat overlying dated sample contains Zone II flora and fauna.

\section{Cledlyn Pingo series, Cardiganshire}

Augered samples from sediment filling collapsed Pingos $\mathrm{M}$ and $\mathrm{U}$, $12 \mathrm{~km}$ W of Lampeter, Cardiganshire $\left(52^{\circ} 05^{\prime} 25^{\prime \prime} \mathrm{N}, 04^{\circ} 14^{\prime} 20^{\prime \prime} \mathrm{W}\right.$, Grid Ref SN 467477). Coll April 1973 and subm by Edward Watson, Dept Geog, Univ Coll Wales, Aberystwyth.

Sample (a) after alkali pretreatment, (b) humate extract.

\section{Birm-485.}

Pingo M, W6, Bed 4 from Boreholes A-D.

Birm-486.

Pingo M, W6, Bed 5 from Boreholes A and B.

\section{Birm-487.}

Pingo M, W6, Bed 5 from Boreholes C and D.

\section{Birm-488.}

Pingo U, W5, Bottom $5 \mathrm{~cm}$ of Bed 4, Boreholes a and b. (a) $8980 \pm 450$ $7030 \mathrm{BC}$

$\delta^{13} C=-24.6 \%$

(b) $9550 \pm 290$ $7600 \mathrm{BC}$

$\delta^{13} C=-27.3 \%$

(a) $9760 \pm 230$ $7810 \mathrm{BC}$

$\delta^{13} C=-25.8 \%$

(b) $9420 \pm 190$ 7470 BC

$\delta^{13} C=-27.0 \%$ o

(a) $8760 \pm 310$ $6810 \mathrm{BC}$

$\delta^{13} \mathrm{C}=-25.3 \%$

(b) $9470 \pm 190$ 7520 BC

$\delta^{13} \mathrm{C}=-28.1 \%$

(a) $9630 \pm 240$

$7680 \mathrm{BC}$

$\delta^{13} \mathrm{C}=-27.1 \%$

(b) $10,060 \pm 380$

8110 BC

$\delta^{13} C=-27.4 \%$ 


\section{Birm-489.}

Pingo U, W5, Bottom $5 \mathrm{~cm}$ of Bed 4, Boreholes $\mathrm{c}$ and d.

General Comment: small samples and thus standard deviations high. Some inconsistency between ages, probably due to contamination in sampling. Dates suggest that organic sedimentation started at Pingo $U$ at about junction of Pollen Zones III and IV and in Pingo M, slightly later. Previous dates from same group of pingos in R, 1973, v 15, p 460-461. For description of these pingos see Watson, 1971, p 382-388, and Watson \& Watson, 1972, p 213-217.

\section{Thurmaston series, Leicestershire}

Vegetable matter from gravel pit at Thurmaston, Leicestershire. Coll 1973 and subm by Andrew Mathieson, Leicester Mus.

\section{Birm-499.}

$$
\begin{gathered}
4220 \pm 130 \\
2270 \mathrm{BC} \\
\delta^{13} C=-27.3 \%
\end{gathered}
$$

Unid. wood from ca $3 \mathrm{~m}$ deep overlain by $.8 \mathrm{~m}$ coarse gravel, $.5 \mathrm{~m}$ mud, sand and gravel and $1.6 \mathrm{~m}$ brown clay $\left(52^{\circ} 41^{\prime} 15^{\prime \prime} \mathrm{N}, 01^{\circ} 06^{\prime} 15^{\prime \prime} \mathrm{W}\right.$, Grid Ref SK 60501041).

\section{Birm-500.}

$$
\begin{aligned}
& 3720 \pm 130 \\
& 1770 \mathrm{BC}
\end{aligned}
$$

$\delta^{13} C=-26.0 \%$

Peat from bed 1.55 to $1.92 \mathrm{~m}$ deep overlying sand and gravel and underlying $1.55 \mathrm{~m}$ clay $\left(52^{\circ} 41^{\prime} 00^{\prime \prime} \mathrm{N}, 01^{\circ} 06^{\prime} 10^{\prime \prime} \mathrm{W}\right.$, Grid Ref SK 60611007). Comment: dates encompass deposition of coarse gravel and antedate formation of highest clay in alluvium of $\mathrm{R}$ Soar.

\section{Birm-501. Long Eaton, Derbyshire}

$7850 \pm 130$ 5900 BC

$$
\delta^{13} \mathrm{C}=-25.7 \%
$$

Wood (? Salix) from large tree trunk, ca $8.5 \mathrm{~m}$ long and $4 \mathrm{~m}$ circum, at 3.05 to $3.35 \mathrm{~m}$ deep overlying gravel at least $3 \mathrm{~m}$ thick at gravel quarry on R Trent flood plain SE of Long Eaton, Derbyshire $\left(52^{\circ} 53^{\prime} 15^{\prime \prime} \mathrm{N}\right.$, $01^{\circ} 14^{\prime} 40^{\prime \prime} \mathrm{W}$, Grid Ref SK 50953301). Coll Jan 1974 and subm by G R Suffield, Ready Mixed Concrete Ltd, Nottingham. Comment: dates alluviation of one of Britain's major rivers. 
Aston Mill series, Worcestershire

(a)

$\mathbf{2 9 , 5 0 0}$

$+\mathbf{1 7 0 0}$

\section{$27,550 \mathrm{BC}$}

Birm-504. Field 3, Silt 9

(b) $>\mathbf{1 8 , 8 0 0}$

$\delta^{13} \mathrm{C}=-24.9^{\circ} \%$

$\delta^{13} \mathrm{C}=-22.4 \%$

Plant material washed from silt pocket on Lias clay, covered by ca $2 \mathrm{~m}$ bedded limestone gravel and ca $1 \mathrm{~m}$ loam near Aston Mill, Worcestershire $\left(52^{\circ} 00^{\prime} 50^{\prime \prime} \mathrm{N}, 02^{\circ} 04^{\prime} 45^{\prime \prime} \mathrm{W}\right.$, Grid Ref SO 94533505). Coll Aug 1972 and subm by P F Whitehead, Dept Geol, Univ Birmingham. Sample (a) after alkali pretreatment, (b) humate extract.

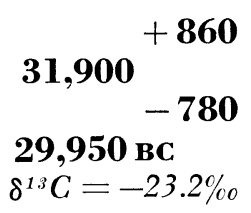

Birm-505. Field 5, Silt 26

$\delta^{13} \mathrm{C}=-23.2 \%$

Moss washed from silt at base of ca $5.5 \mathrm{~m}$ bedded sand and gravel overlain by ca $1 \mathrm{~m}$ loam near Aston Mill, Worcestershire $\left(52^{\circ} 01^{\prime} 15^{\prime \prime} \mathrm{N}\right.$, $02^{\circ} 05^{\prime} 00^{\prime \prime} \mathrm{W}$, Grid Ref SO 94153553). Coll Dec 1973 and subm by PFW. General Comment: dates consistent with each other and with Birm-382; $26,000 \pm 300(\mathrm{R}, 1974, \mathrm{v} 16, \mathrm{p} 286)$ and with Birm-293; 27,650 $\pm 250(\mathrm{R}$, 1973 , v 15, p 5) from same terrace at Beckford. Confirms correlation of terrace with Avon No. 2.

\section{Birm-526. Wick, Worcestershire \\ $4050 \pm 140$ \\ $2100 \mathrm{BC}$ \\ $\delta^{13} \mathrm{C}=-27.0 \%$ o}

Carbonized roots from bed of silty sand, $.3 \mathrm{~m}$ thick, below $4.3 \mathrm{~m}$ ochreous gravel with flint and Bunter pebbles, and above .4m of similar gravel on Lias clay exposed in pipe trench SE of Wick, Worcestershire $\left(52^{\circ} 05^{\prime} 50^{\prime \prime} \mathrm{N}, 02^{\circ} 02^{\prime} 05^{\prime \prime} \mathrm{W}\right.$, Grid Ref SO 97604450). Coll April 1974 and subm by PFW. Comment (FWS): gravel previously assigned to Avon No. 3 terrace (Ipswichian) by Tomlinson (1925), confirmed by presence of Hippopotamus. Dated material must be Flandrian intrusive roots.

Birm-506. Speeton, Yorkshire

$$
\begin{gathered}
11,380 \pm 260 \\
9430 \mathrm{BC} \\
\delta^{13} \mathrm{C}=-28.0 \%
\end{gathered}
$$

Peat with twigs (? Betula and ? Alnus) from 2.59 to $2.64 \mathrm{~m}$ deep (ca +40.03 to $+40.08 \mathrm{~m}$ alt) from sec in upper part of coastal cliff at Speeton, N Yorkshire $\left(54^{\circ} 09^{\prime} 50^{\prime \prime} \mathrm{N}, 00^{\circ} 15^{\prime} 00^{\prime \prime} \mathrm{W}\right.$, Grid Ref TA 145760). Coll Feb 1974 by C A Edwards; subm by L. F Penny, Dept Geol, Univ Hull. Comment: confirms expected Zone II age, similar to Birm-345; 11,260 \pm 100 (R, 1973, v 15, p 459) from Grimston Hall, E Yorkshire. 


\section{Silver Flowe series, Kirkcudbrightshire, Scotland}

Ombrotrophic peat consisting mainly of Sphagnum, Eriophorum, and Calluna, id by P D Hulme, from pool system of Craigeazle bog, Silver Flowe Nature Reserve, Kirkcudbrightshire, Scotland $\left(55^{\circ} 05^{\prime} \mathrm{N}\right.$, 04 ${ }^{\circ} 3^{\prime}$ W, Grid Ref NX 477812). Coll April 1973 and subm by P D Hulme, Dept Pedol, Macaulay Inst Soil Res, Aberdeen.

\section{Birm-507. Site 1}

(a) $1360 \pm 280$ AD 590

$\delta^{13} \mathrm{C}=-23.2 \%$

(b) $1610 \pm 110$ AD 340

$\delta^{13} C=-26.3 \%$

Sample (a) after alkali pretreatment, (b) humate extract from .70 to $.75 \mathrm{~m}$ deep. Comment (PDH): dates formation of pool system of Area B of Craigeazle bog.

(a) $860 \pm 130$ AD 1090

$\delta^{13} C=-23.8 \%$

\section{Birm-508. Site 3}

(b) $920 \pm 130$

AD 1030

$\delta^{13} C=-24.8 \%$

Sample (a) after alkali pretreatment, (b) humate extract from .70 to $.75 \mathrm{~m}$ deep. Comment (PDH): dates formation of pool system of Area AE of Craigeazle bog. Birm-443: $910 \pm 150(\mathrm{R}, 1974, \mathrm{v} 16, \mathrm{p} 291)$ dates start of major phase of pool system development.

\section{Epping Forest series, Essex}

Plant material from recent (sub-Boreal/sub-Atlantic) shallow peat bog $100 \mathrm{~m} \mathrm{~N}$ of junction of Lodge Rd and All main rd in Epping Forest, Essex $\left(51^{\circ} 41^{\prime} \mathrm{N}, 00^{\circ} 04^{\prime} \mathrm{E}\right.$, Grid Ref TL 432999). Coll June 1974 by $\mathrm{P}$ M Oxford; subm by C A Baker, Dept Geog, King's Coll, London.

Birm-525.

$$
\begin{gathered}
\text { (a) } 4250 \pm 120 \\
\mathbf{2 3 0 0} \mathrm{BC} \\
\delta^{13} C=-26.8 \% \\
\text { (b) } 4290 \pm \mathbf{1 0 0} \\
\mathbf{2 3 4 0} \mathrm{BC} \\
\delta^{13} C=-26.8 \%
\end{gathered}
$$

Peat with wood from Borehole B4, 150 to $170 \mathrm{~cm}$ deep. (a) and (b) are independent determinations of the same sample gas in different counters at different filling pressures.

Birm-582.

$$
\begin{aligned}
& 1110 \pm 160 \\
& \text { AD 840 } \\
& \delta^{1 s} C=-26.4 \% o
\end{aligned}
$$

Plant material washed from detritus clay-mud containing Juncus 
and wood fragments from Borehole B4, 95 to $115 \mathrm{~cm}$ deep. Comment: dates Tilia clearance and expansion of Fagus-Carpinus.

General Comment: samples secured by multiple shots with Hiller auger. Sufficient material for Birm-582 only obtained by washing large quantity of sediment from $20 \mathrm{~cm}$ band, $10 \mathrm{~cm}$ either side of Tilia decline level.

\section{Birm-527. Easington, E Yorkshire}

Collagen from antler of Cervus elaphus, id by B Latham, Hull Mus, from within Scrobicularia clay at $-.61 \mathrm{~m}$ alt directly overlying till at Easington, E Yorkshire $\left(53^{\circ} 38^{\prime} 15^{\prime \prime} \mathrm{N}, 00^{\circ} 07^{\prime} 00^{\prime \prime} \mathrm{E}\right.$, Grid Ref TA 411182). Coll Nov 1973 and subm by J F D Bridger, Coll Educ, Hull. Comment (JFDB): antler shows unmistakable evidence of working by Man. Surface of cut portion indicates use of a metal implement verified by Bronze age date. Sample was almost completely enclosed by clay; previous bone finds in area recorded from peat (Bisat, 1952).

\section{Birm-528. Barmston, Yorkshire}

$$
\begin{gathered}
\mathbf{5 7 2 0} \pm \mathbf{1 9 0} \\
\mathbf{3 7 7 0} \mathbf{\text { BC }} \\
\delta^{13} C=-24.0 \%
\end{gathered}
$$

Plant material washed from organic rich silt ca $1.10 \mathrm{~m}$ below cliff top at Barmston, E Yorkshire $\left(54^{\circ} 00^{\prime} 40^{\prime \prime} \mathrm{N}, 00^{\circ} 12^{\prime} 40^{\prime \prime} \mathrm{W}\right.$, Grid Ref TA 174584). Coll Nov 1973 and subm by JFDB. Comment (JFDB \& FWS): sample from contorted lens of silt in stony head, overlying Drab Till. Assuming head to be late Devensian, probably Zone III, sample was expected to be Zone II (ca 11,000). Assoc insect fauna confirms this age. Sample, therefore, must be grossly contaminated.

\section{Birm-529. Catcott Burtle, Somerset \\ $4340 \pm 120$ 2390 BC \\ $\delta^{18} C=-23.8 \%$}

Wood, Quercus, from base of brown clay with stones, foraminifera and occasional mollusks at $1.50 \mathrm{~m}$ deep, underlying $.7 \mathrm{~m}$ sand and overlying weathered Lias clay at Hill Farm, Catcott Burtle, Somerset $\left(51^{\circ}\right.$ $10^{\prime} 55^{\prime \prime} \mathrm{N}, 02^{\circ} 51^{\prime} 38^{\prime \prime} \mathrm{W}$, Grid Ref ST 399430). Coll April 1974 and subm by F W Shotton. Comment (FWS): sequence thought to be interglacial Burtle Beds until determination of date of $4280 \pm 70$ (HAR-463, unpub) on ? Corylus $\log$ at $1.50 \mathrm{~m}$ in earlier excavation (Kidson et al, 1974). New date confirms Flandrian age of sand and clay and requires Flandrian estuarine transgression against hill of Burtle Beds.

\section{Topsham series, Devonshire}

Wood (? Quercus) from excavation of coffer dam on narrow strip of partially reclaimed land on $\mathrm{E}$ edge of tidal $\mathrm{R}$ Exe at Topsham, Devonshire (50 $30^{\prime} 30^{\prime \prime} \mathrm{N}, 00^{\circ} 05^{\prime} 10^{\prime \prime} \mathrm{W}$, Grid Ref SX 962880). Coll April 1974 by R A Cullingford; subm by Allan Straw, Dept Geog, Univ Exeter. 
Birm-533. W2

$$
\begin{gathered}
3910 \pm 130 \\
1960 \mathrm{BC} \\
\delta^{13} \mathrm{C}=-24.6 \%
\end{gathered}
$$

Outermost part of horizontal log, at least $2 \mathrm{~m}$ long and $20 \times 15 \mathrm{~cm}$ at thickest point, with bark intact, at ca $-3.66 \mathrm{~m}$ alt on gravel bed and enclosed by undisturbed water-laid sediments.

\section{Birm-534. W3}

$$
\begin{gathered}
3300 \pm 120 \\
1350 \text { BC } \\
\delta^{13} C=-26.7 \% 0
\end{gathered}
$$

Part of large piece of wood, ca $15 \times 18 \mathrm{~cm}$, from clayey grit layer $6 \mathrm{~cm}$ thick containing much brashy wood, $60 \mathrm{~cm}$ above Birm-533.

General Comment: if sediment is correctly interpreted as non-marine, sea level is unexpectedly low at these dates.

\section{Birm-546. Cahersiveen, County Kerry, Ireland}

$$
\begin{gathered}
2070 \pm 100 \\
120 \text { BC } \\
\delta^{13} C=-25.9 \%
\end{gathered}
$$

Peat from ca $1 \mathrm{~m}$ deep underlying fairly rounded pebbles in sandy matrix from sand pit at Coonanna Sta $\mathrm{E}$ of Cahersiveen, Iveragh Peninsula, Co Kerry, Ireland ( $51^{\circ} 58^{\prime} \mathrm{N}, 10^{\circ} 31^{\prime} \mathrm{W}$, Irish Grid Ref V 4881). Coll Aug 1973 and subm by Irene Benner, Dept Geog, Univ Coll Dublin. Comment: white rootlets within sample were impossible to remove completely. Acid pretreatment only.

\section{Llyn Peris series, Caernarvonshire}

Wood and peat coll by shell and auger boring from Llyn Peris peninsula, Caernarvonshire. Coll March/April 1974 by H Tinsley; subm by E Derbyshire, Dept Geog, Univ Keele.

\section{Birm-554. Ep 14.32}

$$
\begin{gathered}
9400 \pm 220 \\
7450 \text { BC } \\
\delta^{13} C=-23.8 \% 0
\end{gathered}
$$

Peat from 32.08 to $32.15 \mathrm{~m}$ deep, overlying varved clay at SE end of

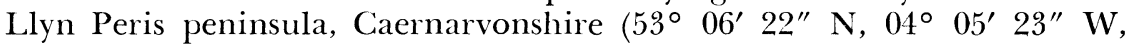
Grid Ref SH 601587). Sample from base of peat $20 \mathrm{~m}$ thick overlain by ca $12 \mathrm{~m}$ gravel. Comment (ED): pollen analysis shows Zone VI/V assemblage.

\section{Birm-555. Ph 6.44}

$11,260 \pm 220$ 9310 BC

$$
\delta^{1.3} \mathrm{C}=-25.7 \%
$$

Peat from lens $50 \mathrm{~cm}$ thick at 18.20 to $18.70 \mathrm{~m}$ deep lying on laminated silt and overlain by gravel from alluvial fan at NW end of Llyn Peris peniinsula, Caernarvonshire $\left(53^{\circ} 07^{\prime} 00^{\prime \prime} \mathrm{N}, 04^{\circ} 06^{\prime} 50^{\prime \prime} \mathrm{W}\right.$, Grid Ref SH 583598. Comment (ED): pollen analysis shows Zone I/II assemblage. 
Birm-556. Ep 11.12

$1750 \pm 100$ AD 200

$\delta^{13} \mathrm{C}=-24.0 \%$

Wood extracted from peat, 12.05 to $12.25 \mathrm{~m}$ deep, underlying ca $12 \mathrm{~m}$ gravel at SE end of Llyn Peris peninsula, Caernarvonshire (53 $06^{\prime} 22^{\prime \prime}$ $\mathrm{N}, 04^{\circ} 05^{\prime}$ 25" W, Grid Ref SH 601587). Comment (ED): pollen analysis shows Zone VIIb assemblage.

General Comment: dates refer to history of sedimentation following deglaciation.

\section{Birm-590. Farmoor, Oxfordshire}

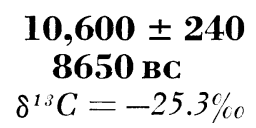

Sticks washed from organic silt ca $1.83 \mathrm{~m}$ deep in gravel ca $3 \mathrm{~m}$ thick, overlying Oxford Clay at Farmoor, Oxfordshire $\left(51^{\circ} 45^{\prime} \mathrm{N}, 01^{\circ} 21^{\prime} \mathrm{W}\right.$, Grid Ref SP 452059). Coll Aug 1974 by C W Berg; subm by G R Coope, Dept Geol, Univ Birmingham. Comment (GRC): organic deposit contains suite of Coleoptera characteristic of Pollen Zone III of which 2 are now exclusively E Siberian species.

\section{B. Other Countries}

\section{Birm-490. Iles Crozet, French Sub-Antarctic Territories}

(a) $\delta^{14} \mathbf{C}=+\mathbf{6 3 . 1} \pm \mathbf{5 1 . 5} \%$ Modern $\delta^{13} C=-19.5 \%$

$$
\begin{array}{r}
290 \pm 100 \\
\text { AD } 1660 \\
\delta^{13} C=-23.2 \%
\end{array}
$$

Organic silt ca $30 \mathrm{~cm}$ thick with rootlets overlain by ca $2.2 \mathrm{~m}$ coarse gravel with large stones (ca $15 \mathrm{~cm}$ long) in small valley $.5 \mathrm{~km} \mathrm{~S}$ of Port Alfred Base, Ile de la Possession, Iles Crozet, French Sub-Antarctic Territories $\left(46^{\circ} 26^{\prime} \mathrm{S}, 51^{\circ} 52^{\prime} \mathrm{E}\right)$. Coll Jan 1973 and subm by Lewis Davies, Dept Zool, Univ Durham. Comment: rootlets (a) picked out and treated with acid only, remaining soil treated with $0.5 \% \mathrm{NaOH}$ and solution precipitated with HCl, (b). Silt from same bed contains high Lycopod pollen. Date indicates recent origin.

\section{Okstindan series, Norway}

Organic material from Neoglacial end moraines of Okstindan glaciers, Norway. Coll Aug - Sept 1973 and subm by N J Griffey, Dept Geol, Univ Reading.

\section{Birm-492.}

$$
\begin{gathered}
2000 \pm 110 \\
50 \mathrm{BC} \\
\delta^{1.3} C=-25.4 / / \mathrm{C}
\end{gathered}
$$

Peat from $3.3 \mathrm{~m}$ deep in outer end moraine of $\mathrm{N}$ lobe of Austre Okstindbreen glacier ca $10 \mathrm{~m} \mathrm{~S}$ of outwash river $\left(66^{\circ} 02^{\prime} 30^{\prime \prime} \mathrm{N}, 14^{\circ} 23^{\prime}\right.$ $\left.00^{\prime \prime} \mathrm{E}\right)$. 


\section{Birm-493.}

$4790 \pm 120$

2840 BC

$\delta^{13} C=-24.6 \%$ o

Peat from $1.75 \mathrm{~m}$ deep in inner end moraine of $\mathrm{E}$ lobe of Austre Okstindbreen glacier immediately $\mathrm{S}$ of outwash river $\left(66^{\circ} 02^{\prime} 30^{\prime \prime} \mathrm{N}, 14^{\circ}\right.$ $23^{\prime} 00^{\prime \prime} \mathrm{E}$ ).

Birm-494.

(a) $8030 \pm 170$ $6080 \mathrm{BC}$

$\delta^{13} \mathrm{C}=-25.8 \%$

(b) $8480 \pm 170$ $6530 \mathrm{BC}$

$\delta^{13} C=-26.1 \%$ o

Peat (a) after alkali pretreatment, (b) humate extract from $1.55 \mathrm{~m}$

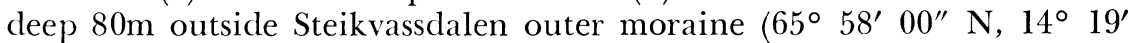
$\left.00^{\prime \prime} \mathrm{E}\right)$. Comment: peat formed shortly after deglaciation of region.

Birm-495.

(a) $2090 \pm 110$

140 BC

$\delta^{13} \mathrm{C}=-25.2 \%$

(b) $1780 \pm 110$ AD 170

$\delta^{13} C=-26.5 \%$

Peat (a) after alkali pretreatment, (b) humate extract from $1.35 \mathrm{~m}$ deep on $\mathrm{E}$ side of outermost end moraine of $\mathrm{N}$ lobe of Austre Okstindbreen $\left(66^{\circ} 03^{\prime} 00^{\prime \prime} \mathrm{N}, 14^{\circ} 21^{\prime} 00^{\prime \prime} \mathrm{E}\right)$.

(a) $1600 \pm 120$ AD 350

$\delta^{13} C=-23.3 \%$

Birm-496.

(b) $1500 \pm 120$ AD 450

$\delta^{13} C=-23.1 \%$

Peat (a) after alkali pretreatment, (b) humate extract from $1.80 \mathrm{~m}$ deep in end moraine of Corneliussensbreen glacier on outside of $\mathrm{S}$ moraine ridge sequence $\left(66^{\circ} 00^{\prime} 30^{\prime \prime} \mathrm{N}, 14^{\circ} 24^{\prime} 00^{\prime \prime} \mathrm{E}\right)$.

Birm-497.

$\mathbf{2 4 0} \pm \mathbf{1 0 0}$
AD 1710
$\delta^{13} C=-26.0 \%$

$\mathrm{NaOH}$ soluble fraction of $\mathrm{A}_{\mathrm{f}-\mathrm{h}}$ horizon of podsol from $1.09 \mathrm{~m}$ deep in inner end moraine of Steikvassbreen glacier at base of valley $\left(65^{\circ} 58^{\prime} 00^{\prime \prime}\right.$ $\mathrm{N}, 14^{\circ} 19^{\prime} 00^{\prime \prime} \mathrm{E}$ ).

Birm-498.

$3170 \pm 120$

$1220 \mathrm{BC}$

$\delta^{13} C=-26.2 \%$

$\mathrm{NaOH}$ soluble fraction of $\mathrm{A}_{\mathrm{f}-\mathrm{h}}$ horizon of podsol from $1.30 \mathrm{~m}$ deep in outer end moraine of Steikvassbreen glacier at base of valley $\left(65^{\circ} 58^{\prime}\right.$ $00^{\prime \prime} \mathrm{N}, 14^{\circ} 19^{\prime} 00^{\prime \prime} \mathrm{E}$ ). 
General Comment: Birm-492, -493, -495, and -496 antedate formation of end moraines. Birm-497 and-498 dates are maximum of 2 end moraines. Other dates from same area in Okstindan Research Proj, Rept 5 (Alexander \& Worsley, 1973).

\section{Birm-522. Three Creeks, W Minnesota}

$$
\begin{array}{r}
>\mathbf{4 6 , 9 0 0} \\
\delta^{13} C=-24.8 \% \text { \% }
\end{array}
$$

Unid. wood near base of clay/loam layer 17.98 to $25.9 \mathrm{~m}$ deep at Three Creeks, Red Lake Falls, $1.93 \mathrm{~km} \mathrm{~W}$ and $1.13 \mathrm{~km} \mathrm{~N}$ of courthouse at cutback on S side of Red Lake R, W Minnesota (47. $53^{\prime} \mathrm{N}, 96^{\circ} 18^{\prime} \mathrm{W}$ ). Coll July 1973 by S R Moran; subm by A C Ashworth, Dept Geol, North Dakota State Univ. Comment: date is minimum for coleopterous assemblage within same horizon as sample, of I-5317: >39,900, and GSC-1666: $>39,000$ (unpub) from similar horizon.

\section{Birm-523. Arthur's Pass, S Island, New Zealand}

$8960 \pm 140$ 7010 BC

$\delta^{13} \mathrm{C}=-27.3 \%$

Phyllocladus wood peat from basal $12 \mathrm{~cm}$ of $3.5 \mathrm{~m}$ sequence of organic peat and silt overlying inorganic silt and sand of glaciofluvial (?) provenance in bog W of Arthur's Pass on W side of $\mathrm{Rd} 73$ at $+914 \mathrm{~m}$ alt in S I, New Zealand (42 $\left.54^{\prime} 30^{\prime \prime} \mathrm{S}, 171^{\circ} 33^{\prime} 30^{\prime \prime} \mathrm{E}\right)$. Coll Jan 1974 and subm by M R Kelly, Dept Environmental Sci, Univ Lancaster. Comment (MRK): site lies immediately behind major moraine loop which is part of important sequence of late glaciation and neoglacial moraines of $S$ Alps. Date is minimum for glacial event.

\section{Maktak Fjord series, Baffin Island}

Moss peat from bed $1.07 \mathrm{~m}$ thick in terminal moraine of Maktak Glacier, Baffin I $\left(67^{\circ} 30^{\prime} \mathrm{N}, 63^{\circ} 20^{\prime} \mathrm{E}\right)$. Coll 1972 and subm by $\mathrm{G} \mathrm{S}$ Boulton, Dept Environmental Studies, Univ E Anglia.

\section{Birm-535. Horizon B}

$$
\begin{gathered}
1970 \pm 200 \\
20 \text { BC } \\
\delta^{13} C=-24.5 \%
\end{gathered}
$$

From 24 to $26 \mathrm{~cm}$ below top of peat bed.

\section{Birm-536. Horizon C}

$$
\begin{aligned}
& 2240 \pm 190 \\
& 290 \text { BC }
\end{aligned}
$$

From 51 to $53 \mathrm{~cm}$ below top of peat bed.

General Comment: samples from same sec as Birm-370: $1480 \pm 160$ at 0 to $5 \mathrm{~cm}$ below top of peat bed and Birm-380: $2500 \pm 170$ at 88 to $100 \mathrm{~cm}$ (R, 1973, v 15, p 463).

\section{Papua series, New Guinea}

Lake mud obtained using Mackereth mini-corer from $\mathrm{W}$ and $\mathrm{S}$ Highlands of Papua, New Guinea. Coll July 1973 and subm by F Oldfield, School of Independent Studies, Univ Lancaster. 
Birm-587. Lake Ipea, Sirunki, W Highlands

Core 3,52 to $56 \mathrm{~cm}\left(05^{\circ} 25^{\prime} \mathrm{S}, 143^{\circ} 30^{\prime} \mathrm{E}\right)$.

Birm-588. Lake Ipea, Sirunki, W Highlands

Core 3,78 to $82 \mathrm{~cm}\left(05^{\circ} 25^{\prime} \mathrm{S}, 143^{\circ} 30^{\prime} \mathrm{E}\right)$.

\section{Birm-589. Lake Egari, S Highlands}

Core 4,90 to $94 \mathrm{~cm}\left(06^{\circ} 12^{\prime} \mathrm{S}, 143^{\circ} 40^{\prime} \mathrm{E}\right)$.

General Comment: acid pretreatment only.
$1220 \pm 150$

AD 730

$\delta^{1.3} C=-23.3 \%$

$5140 \pm 110$

3190 BC

$\delta^{13} \mathrm{C}=-17.9 \%$

$1850 \pm 100$

AD 100

$\delta^{13} C=-24.6 \%$

II. ARCHAEOLOGIC SAMPLES

A. British Isles

\section{Iron Age hill fort series, Surrey}

Charcoal from neighbouring Iron age hill forts in Surrey. Coll and subm by F H Thompson, Soc Antiquaries, Burlington House, London.

Birm-468. Anstiebury

$$
\begin{gathered}
2380 \pm 120 \\
430 \mathrm{BC} \\
\delta^{13} C=-26.5 \%
\end{gathered}
$$

Sample from post hole in greensand ca $4.27 \mathrm{~m} \mathrm{~S}$ of $\mathrm{N}$ butt end of main ditch at $\mathrm{NE}$ entrance of fort at Anstiebury, near Dorking, Surrey $\left(51^{\circ} 11^{\prime} \mathrm{N}, 00^{\circ} 21^{\prime} \mathrm{W}\right.$, Grid Ref TQ 155440). Coll July 1973.

\section{Birm-591. Holmbury Hill}

$$
\begin{gathered}
2260 \pm 100 \\
310 \text { BC } \\
\delta^{13} C=-21.8 \%
\end{gathered}
$$

Sample assoc with sherds of Iron age pottery from $.9 \mathrm{~m}$ diam hearth of reddened stone set in small pit $30 \mathrm{~m} \mathrm{E}$ inside main rampart at Holmbury Hill, Surrey $\left(51^{\circ} 10^{\prime} \mathrm{N}, 00^{\circ} 25^{\prime} \mathrm{W}\right.$, Grid Ref TQ 104430). Coll Aug 1974. Comment: area $2.4 \times 2.4 \mathrm{~m}$ excavated to check anomaly located during fluxgate gradiometer survey of interior of hill fort. Previous evidence (Wimbolt, 1930, p 156) indicated Early Iron age occupation of La Tène III period.

General Comment: pre-Roman Iron age dates.

\section{Skara Brae series, Orkney Islands, NE Scotland}

Collagen from bone samples (Bos longifrons) of Neolithic site on $\mathrm{S}$ edge of Bay of Skaill at Skara Brae, Orkney Is off NE Scotland $\left(59^{\circ} 02^{\prime}\right.$ $50^{\prime \prime} \mathrm{N}, 03^{\circ} 20^{\prime} 40^{\prime \prime} \mathrm{W}$, Grid Ref HY 231187). Coll July-Aug 1973 and subm by D V Clarke, Natl Mus Antiquaries, Edinburgh, Scotland. 


\section{Birm-477. Trench 2, Sec B, S13A}

$3950 \pm 100$

2000 BC

$\delta^{13} \mathrm{C}=-33.6 \%$

From ca 2.83 to $2.96 \mathrm{~m}$ deep. Comment: anomalously low ${ }^{13} \mathrm{C}$ concentration possibly due to incomplete conversion to methane or contamination by $\mathrm{N}_{2} \mathrm{O}$.

\section{Birm-478. Trench 2, Sec B, S13B}

$$
\begin{gathered}
3850 \pm 140 \\
1900 \text { BC } \\
\delta^{13} C=-22.8 \%
\end{gathered}
$$

From ca 2.83 to $2.96 \mathrm{~m}$ deep. Comment (DVC): both Birm-477 and -478 from earliest phase of main activity on site. Dates commencement of construction.

\section{Birm-480. Trench 2, Sec B, S18B}

$$
\begin{gathered}
4320 \pm 100 \\
2370 \text { BC } \\
\delta^{13} C=-21.3 \%
\end{gathered}
$$

From ca 3.53 to $3.66 \mathrm{~m}$ deep. Comment (DVC): assoc with earliest human activity on site. Possibly represents preparations for construction. General Comment: previous dates from this site are Birm-433 to -438 (R, 1974 , v 16, p 300-301). Early excavations including numerous animal remains described by Stewart (1914).

\section{Birm-491. Moycraig, Antrim, Northern Ireland}

$$
\begin{aligned}
& \mathbf{1 0 0 0} \pm \mathbf{1 1 0} \\
& \mathbf{A D} 950 \\
& \delta^{13} \mathrm{C}=-25.7 \%
\end{aligned}
$$

Wood (? Quercus) from horizontal wheel assembly of mill from Moy-

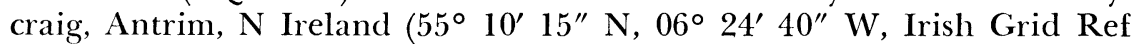
D 015365). Originally coll 1850 (MacAdam, 1856) subsequently coll Dec 1973 from Ulster Mus and subm by P A Rahtz, Hist School, Univ Birmingham. Comment: only horizontal wheel assembly in NW Europe; age is important in the history of milling.

\section{Fisherwick series, Staffordshire}

Charcoal from archaeologic excavation at Fisherwick, Staffordshire $\left(01^{\circ} 44^{\prime} 10^{\prime \prime} \mathrm{N}, 52^{\circ} 40^{\prime} 10^{\prime \prime} \mathrm{W}\right.$, Grid Ref SK 17880826). Coll Sept 1973 and subm by Christopher Smith, Dept Archaeol, Univ Nottingham.

\section{Birm-502. F12}

Comment: dates assoc pottery.

Birm-503. F53

Comment: dates assoc timber structure.

$$
\begin{gathered}
2800 \pm 140 \\
850 \text { BC } \\
\delta^{13} C=-24.9 \%
\end{gathered}
$$

$3120 \pm 140$

$1170 \mathrm{BC}$

$\delta^{1.3} \mathrm{C}=-26.2 \%$ 
Birm-524. Tamworth, Staffordshire

$$
\begin{array}{r}
\mathbf{5 7 0} \pm \mathbf{1 0 0} \\
\text { AD } 1380 \\
\delta^{13} C=-24.5 \% \text { o }
\end{array}
$$

Wood, unid., 2.1m deep, from timber structure consisting of an upright and horizontal member apparently supporting platform $30 \mathrm{~m}$ from present $\mathrm{N}$ bank of river at Ankerside, Tamworth, Staffordshire $\left(52^{\circ} 37^{\prime}\right.$ $50^{\prime \prime} \mathrm{N}, 01^{\circ} 41^{\prime} 30^{\prime \prime} \mathrm{W}$, Grid Ref SK 20890392). Coll Feb 1974 by $\mathrm{K}$ Sheridan; subm by P A Rahtz. Comment: dates structure. Horn cores together with one fragment of medieval pottery and one medieval shoe sole found assoc with structure.

\section{Wrekin hill fort series, Shropshire}

Charcoal and carbonized grain from latest hut post holes of late Bronze or Iron age hill fort, Wrekin, Shropshire $\left(52^{\circ} 40^{\prime} \mathrm{N}, 02^{\circ} 33^{\prime}\right.$ W, Grid Ref SJ 631083). Coll May 1973 and subm by S C Stanford, Dept Extramural Studies, Univ Birmingham.

\section{Birm-530. F35a S34}

$$
\begin{gathered}
2290 \pm 100 \\
340 \text { BC } \\
\delta^{13} C=-23.8 \% \text { o }
\end{gathered}
$$
Hut 1 .

Wood charcoal, probably part of post, from final post socket of

\section{Birm-531. F30 S13}

Carbonized grain from final post socket of Hut 1.

\section{Birm-532. F16 S11}

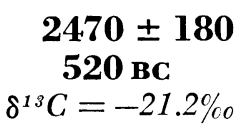

$$
1960 \pm 90
$$$$
10 \mathrm{BC}
$$$$
\delta^{13} \mathrm{C}=-22.2 \%
$$

Carbonized grain from final post socket on hut Terrace F8.

General Comment: samples from pre-Roman capital of British tribe of Cornovii prior to transfer to legionary camp at Virconium on $\mathrm{R}$ Severn ca $8 \mathrm{~km} \mathrm{SE}$ of Shrewsbury. Dates could indicate at least 2 periods or a single continuous period of occupation of outer camp.

\section{City of London series}

Samples from ? Saxon contexts within City of London. Subm by Brian Hobley, Guildhall Mus, London.

\section{Birm-547.}

Charcoal from infill of hut pit from Bread St, London. Coll Dec 1973 by M Guttares. Comment: dates rare pottery from hut pit of the "Dark Ages" of British archaeology. 
Birm-548.

Wood, silver birch, from stakes embedded in silt of ? Saxon waterfront $S$ of Lower Thames St, Billingsgate, London $\left(51^{\circ} 30^{\prime} 30^{\prime \prime} \mathrm{N}, 00^{\circ} 05^{\prime}\right.$ 10" W, Grid Ref TQ 32958066). Coll April 1974 by G B Clewley. Comment: dates construction of riverside structure.

\section{Birm-551. Irthlingborough gravel pit, Northamptonshire \\ $430 \pm 330$ AD 1520 \\ $\delta^{13} \mathrm{C}=-20.6 \%$ 。}

Wood $1.83 \mathrm{~m}$ deep underlying river alluvium from part of stake, one of many delimiting stone causeway across valley of $\mathrm{R}$ Nene at Irthlingborough gravel pit, Northamptonshire $\left(52^{\circ} 18^{\prime} 30^{\prime \prime} \mathrm{N}, 00^{\circ} 36^{\prime} 26^{\prime \prime} \mathrm{W}\right.$, Grid Ref SP 949687). Coll Oct 1965 and subm by D N Hall, Northampton Mus. Comment (DNH): unexpectedly late but agrees with other recent evidence for late accumulation of alluvium due to soil wash following opening up of field system in Medieval times. Large standard deviation as sample was very small.

\section{Birm-552. Easton Maudit, Northamptonshire}

$$
\begin{array}{r}
710 \pm 100 \\
\text { AD } 1240 \\
\delta^{13} C=-23.9 \%
\end{array}
$$

Charcoal underlying modern plough soil containing iron slag at Easton Maudit, Northamptonshire $\left(52^{\circ} 11^{\prime} 51^{\prime \prime} \mathrm{N}, 00^{\circ} 41^{\prime} 20^{\prime \prime} \mathrm{W}\right.$, Grid Ref SP 895563). Coll April 1974 and subm by DNH. Comment (DNH): dates final clearance in Medieval times of woodland on high ground between $\mathrm{R}$ Nene and $\mathrm{R}$ Ouse, preceding development of open field system.

\section{Birm-553. Caynham Camp, Ludlow, Shropshire}

$2310 \pm 300$ $360 \mathrm{BC}$

$\delta^{13} C=-21.0 \%$

Charcoal $2.45 \mathrm{~m}$ deep in fill of early ditch of clay and burnt limestone fragments, assoc with timber-laced rampart, replaced by a dump rampart at Caynham Camp $3.22 \mathrm{~km} \mathrm{SE}$ of Ludlow, Shropshire $\left(52^{\circ} 21^{\prime}\right.$ $25^{\prime \prime} \mathrm{N}, 02^{\circ} 39^{\prime} 50^{\prime \prime} \mathrm{W}$, Grid Ref SO 545737). Coll June 1974 and subm by Simon Hillson, Dept Geol, Univ Birmingham. Comment: dates timber-laced rampart. Four different constructional stages of rampart have been observed (Gelling, 1959; 1960; 1961). Large standard deviation due to small sample size. $C f$ other dates on Iron age camp in Welsh Marches at Midsummer Camp, Eastnor, near Ledbury, Herefordshire Birm-142: $2370 \pm 190$ and Birm-143: $2000 \pm 200(\mathrm{R}, 1970, \mathrm{v} 12, \mathrm{p}$ 396) and the Wrekin series (above).

\section{B. Other countries}

Monte Covolo series, Italy

Charcoal from adjacent stratified layers in deposit covering end of 
Neolithic period and beginning of Early Bronze age at Monte Covolo, Brescia, N Italy $\left(45^{\circ} 40^{\prime} \mathrm{N}, 10^{\circ} 38^{\prime} \mathrm{E}\right)$. Coll Aug 1973 and subm by L H Barfield, Dept Ancient Hist \& Archaeol, Univ Birmingham.

\section{Birm-469. Level 4}

From Early Bronze age layer, .50m deep.

\section{Birm-470. Level 6}

From Upper Bell Beaker layer, .75m deep.

\section{Birm-471. Layer 8}

From Lower Bell Beaker layer, .75m deep.

\section{Birm-472. Layer 13}

From Upper Lagozza and Neolithic layer, 1.50m deep.

\section{Birm-473. Layer 20}

From Lower Lagozza and Neolithic layer, 1.70m deep.

General Comment (LHB): chronologic order of dates corresponds with sequence of stratified deposits. Birm-473 agrees with dates from other Lagozza sites, eg, Pi-34: $4794 \pm 90$ (R, 1961, v 3, p 102) and R-337: 4805 $\pm 50(\mathrm{R}, 1968, \mathrm{v} 10$, p 357). Birm-472 is later than estimated age for Lagozza culture but verifies suggestion of Barfield and Fasani (1972) that Lagozza culture survived alongside Copper age Remdello tradition until late in 3rd millennium. Birm-469-471 are within range of available dates for Bell Beaker culture elsewhere in Europe. Unfortunately large standard deviation on these dates does not solve important problem as to whether Bell Beaker phase at Monte Covolo belongs to an early or late stage of development.

\section{Peruvian coastal series}

Samples from prehistoric sites on coast of Peru, S of Lima. Coll 1973 and subm by Frederic Engel, Inst Anthropol Agric Precolumbina, Lima, Peru.

\section{Birm-509. (W 1700) Boca Rio Ica}

$$
\begin{array}{r}
440 \pm 100 \\
\text { AD } 1510 \\
\delta^{13} C=-24.4 \%
\end{array}
$$

Wood (mimosa) from post sustaining wattle and daub house walls in village on high sand dune on $\mathrm{S}$ bank near mouth of $\mathrm{R} \mathrm{Ica}\left(14^{\circ} 47^{\prime} \mathrm{S}, 75^{\circ}\right.$ $\left.33^{\prime} \mathrm{W}\right)$ at $+80 \mathrm{~m}$ alt. Comment: village contains late Ica pottery. 
(a) $5330 \pm 120$ 3380 BC

$\delta^{13} \mathrm{C}=-0.2 \%$

\section{Birm-510. (V 3414) Pampa de los Perros}

(b) $5470 \pm 100$ 3520 BC

$\delta^{1 .} \mathrm{C}=-0.4 \%$

Marine shells sorted from ashes and food remains from small encampment in dry canyon in "lomas" region of central coast of Peru at Pampa de los Perros, Chillon Basin (1 $11^{\circ} 54^{\prime} 20^{\prime \prime} \mathrm{S}, 76^{\circ} 06^{\prime} 44^{\prime \prime} \mathrm{W}$ ) at $+250 \mathrm{~m}$ alt. Comment: (a) inner and (b) middle fraction of shells from Layer 200.

\section{Birm-511. ( V 3398) Boca Rio Ica}

$$
\begin{gathered}
\mathbf{7 5 2 0} \pm \mathbf{3 0 0} \\
\mathbf{5 5 7 0} \text { BC } \\
\delta^{18} \mathrm{C}=-19.5 \% \text { o }
\end{gathered}
$$

Plant remains from large "spots" of refuse including marine shells, bits of cord and feces from Layer 200 under wind blown sand $850 \mathrm{~m} \mathrm{E}$ of coast and $50 \mathrm{~m} \mathrm{~N}$ of river bed on terrace $+35 \mathrm{~m}$ alt at Boca Rio Ica (14 ${ }^{\circ}$ $\left.52^{\prime} \mathrm{S}, 75^{\circ} 34^{\prime} \mathrm{W}\right)$. Comment: dates early occupation at mouth of $\mathrm{R}$ Ica.

\section{Birm-512. (V 3417) Carhuas}

$$
\begin{gathered}
2500 \pm 110 \\
550 \mathrm{BC} \\
\delta^{13} C=-8.7 \% 0
\end{gathered}
$$

Small corn cobs from plant and animal remains in refuse of Level 200 at fishing village on cliff of Carhuas beach $\left(14^{\circ} 12^{\prime} \mathrm{S}, 76^{\circ} 10^{\prime} \mathrm{W}\right)$ at $+20 \mathrm{~m}$ alt. Comment: dates S-most Chavin village. Site yielded polychromic painted cotton garments to treasure hunters. Sample falls into category of plants with high $\delta^{13} \mathrm{C}$ value -6 to $-19 \%$ (Smith \& Epstein, $1971)$, with $\delta^{13} \mathrm{C}$ of $-14.0 \%$ given for Zea mays $\mathrm{L}$.

\section{Birm-513. (V 3395) Mouth of River Ica}

$$
\begin{gathered}
\mathbf{5 4 3 0} \pm 140 \\
\mathbf{3 4 8 0} \mathbf{B C} \\
\delta^{13} C=-19.6 \%
\end{gathered}
$$

Reeds and canes from medium-sized village on terrace of $\mathrm{N}$ bank of $\mathrm{R}$ Ica near its mouth at $+35 \mathrm{~m}$ alt and ca $1.7 \mathrm{~km}$ from coast $\left(14^{\circ} 52^{\prime} \mathrm{S}, 75^{\circ}\right.$ $\left.34^{\prime} \mathrm{W}\right)$. Comment: village yielded beans but not cotton. Obsidian flakes were found within refuse and fine points on surface. Similar village found by submitter $18 \mathrm{~km} \mathrm{~S}$ at mouth of R Nazca (Engel, 1966). Dates advent of bean planters along Peruvian coast.

\section{Birm-514. (V 3386) Bahia Independencia}

(a) $600 \pm 100$ AD 1350

$\delta^{13} C=-0.4 \%$

(b) $420 \pm 120$

AD 1530

$\delta^{13} \mathrm{C}=-0.3 \%$

Marine shells broken by Man covering large area on emerged terrace from $+40 \mathrm{~m}$ to $+80 \mathrm{~m}$ alt at Bahia Independencia $\left(14^{\circ} 08^{\prime} \mathrm{S}, 76^{\circ} 17^{\prime} \mathrm{W}\right)$. 
Comment: wind erosion removed all organic material. (a) inner and (b) middle fraction.

\section{Birm-515. (V 3385) Loma de Paloma}

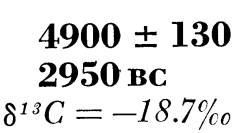

Fish meal from lst refuse layer below wind blown sand, Layer 200, from very large village ( 30 huts and 85 graves in single cut of $500 \mathrm{sq} \mathrm{m}$ ) at Paloma, Chilca Basin ( $12^{\circ} 25^{\prime} 55^{\prime \prime} \mathrm{S}, 76^{\circ} 45^{\prime} 05^{\prime \prime} \mathrm{W}$ ) at $+200 \mathrm{~m}$ alt. Comment: lichens removed by diluted acid before dating. Village belongs to late pre-farming period.

\section{Birm-516. ( V 3368) Loma de Paloma}$$
6410 \pm 140
$$$$
4460 \text { вC }
$$$$
\delta^{13} \mathrm{C}=-1.8 \% \text { o }
$$

Charcoal from hearth inside House XXII whose floor is formed by sterile ground at village of Paloma, Chilca basin $\left(12^{\circ} 25^{\prime} 55^{\prime \prime} \mathrm{S}, 76^{\circ} 45^{\prime}\right.$ $\left.05^{\prime \prime} \mathrm{W}\right)$. Comment: dates pre-farming settlers who built Village 613 .

\section{Birm-517. (V 3416) Pampa de los Perros}

$$
\begin{gathered}
\text { (a) } 6810 \pm 140 \\
\mathbf{4 8 6 0} \mathrm{BC} \\
\delta^{13} C=-1.1 \% \\
\text { (b) } 7 \mathbf{1 5 0} \pm \mathbf{1 4 0} \\
\mathbf{5 2 0 0} \mathrm{BC} \\
\delta^{13} C=-2.0 \%
\end{gathered}
$$

Shells from Level 200 covered by caliche at Pampa de los Perros, Chillon Basin ( $\left.11^{\circ} 54^{\prime} 20^{\prime \prime} \mathrm{S}, 76^{\circ} 06^{\prime} 44^{\prime \prime} \mathrm{W}\right)$ at $+250 \mathrm{~m}$ alt. Comment: (a) inner and (b) middle fraction.

\section{Birm-518. (V 3413) Pampa de los Perros}

$$
\begin{gathered}
6080 \pm 130 \\
4130 \mathrm{BC} \\
\delta^{13} C=-17.9 \% \circ
\end{gathered}
$$

Charcoal from Layer 200, a large refuse mound of ashes and charred wood lying beneath Layer 100 of earth at Pampa de los Perros, Chillon Basin (11 $\left.55^{\prime} 20^{\prime \prime} \mathrm{S}, 76^{\circ} 07^{\prime} 00^{\prime \prime} \mathrm{W}\right)$ at $+160 \mathrm{~m}$ alt.

General Comment: other samples for Arid land Prehistory program of Natl Agrarian Univ Peru dated by Pennsylvania (R, 1974, v 16, p 231232) and Nancy Labs (R, 1974, v 16, p 123).

\section{Parpalló series, Spain}

Collagen from antler of Cervus elaphus from cave deposit at Parpalló, Valencia, Spain $\left(39^{\circ} 00^{\prime} \mathrm{N}, 00^{\circ} 20^{\prime} \mathrm{W}\right)$. Coll 1929 by L Pericot Garcia and stored in Valencia Mus; subm by Ian Davidson, Dept Prehist and Archaeol, Univ New England, NSW.

\section{Birm-519. 30545}

$13,800 \pm 380$

$11,850 \mathrm{BC}$

$\delta^{13} \mathrm{C}=-18.5 \%$

From 1.50 to $1.70 \mathrm{~m}$ deep within Magdalenian III layer at .80 to 
2.50m deep. Cf GrN-1913: 14,160 \pm 80 (R, 1963, v 5, p 169) and M-828: $13,900 \pm 700(\mathrm{R}, 1960, \mathrm{v} 2, \mathrm{p} 46)$.

\section{Birm-520. A}

$20,170 \pm 380$

18,220 BC

$\delta^{13} \mathrm{C}=-17.3 \%$

From 2 grouped spits, 6.25 to $6.50 \mathrm{~m}$ deep and 7.25 to $7.75 \mathrm{~m}$ deep, which are from tops of different archaeologic groups. Layer 6.25 to $7.25 \mathrm{~m}$ described as Lower Solutrean and cultural remains below as Gravettian (Pericot Garcia, 1942). Cf BM-859: 20,490 ${ }_{-800}^{+900}$ (unpub), considered unexpectedly old for Lower Solutrean.

\section{Birm-521. 30550}

$$
\begin{aligned}
& 17,900 \pm 340 \\
& 15,950 \text { BC }
\end{aligned}
$$$$
\delta^{13} \mathrm{C}=-19.0 \%
$$

From 6 to $6.25 \mathrm{~m}$ deep within Middle Solutrean layer at 5.25 to $6.25 \mathrm{~m}$ deep. Comment: 1st date on Solutrean-Gravettian industry.

General Comment: series suggests that Solutrean culture lingered longer in Spain than in France (Davidson, 1974).

\section{GROUND WATER SAMPLES}

\section{Warwick/Stratford series}

Radiocarbon concentrations of dissolved carbonate species in subsurface waters from Keuper Sandstone aquifers in Warwick/Stratfordupon-Avon region are reported as \% of modern without correction for limestone dilution. $\delta^{13} \mathrm{C}$ contents are based on Craig PDB limestone equal to $0.00 \%$. Carbonate species extraction was by precipitation with $\mathrm{BaCl}_{2}$ (IAEA, 1968, p 11). Samples coll July 1974 and subm by J W Lloyd, Dept Geol, Univ Birmingham.

\section{Birm-559. Moreton Morell}

\begin{tabular}{|c|c|c|}
\hline $\begin{array}{l}\text { Birm-560. Thelsford Pumping Sta } \\
\left(52^{\circ} 13^{\prime} 10^{\prime \prime} \mathrm{N}, 01^{\circ} 36^{\prime} 00^{\prime \prime} \mathrm{W} \text {, Grid Ref SP } 273580\right)\end{array}$ & -12.4 & $1.1 \pm 0.3$ \\
\hline $\begin{array}{l}\text { Birm-561. Wellsbourne Pumping Sta } \\
\left(52^{\circ} 11^{\prime} 15^{\prime \prime} \text { N, } 01^{\circ} 36^{\prime} 15^{\prime \prime} \text { W, Grid Ref SP 271543) }\right.\end{array}$ & -13.2 & $2.8 \pm 0.4$ \\
\hline $\begin{array}{l}\text { Birm-562. Alveston Hill Pumping Sta } \\
\left(52^{\circ} 11^{\prime} 20^{\prime \prime} \mathrm{N}, 01^{\circ} 40^{\prime} 15^{\prime \prime} \mathrm{W} \text {, Grid Ref SP } 226544\right)\end{array}$ & -13.7 & $3.2 \pm 0.3$ \\
\hline Flowers Borehole & -12.2 & $0.01 \pm 0.04$ \\
\hline
\end{tabular}

$\left(52^{\circ} 11^{\prime} 40^{\prime \prime} \mathrm{N}, 01^{\circ} 33^{\prime} 20^{\prime \prime} \mathrm{W}\right.$, Grid Ref SP 303553)

$\left(52^{\circ} 11^{\prime} 45^{\prime \prime} \mathrm{N}, 01^{\circ} 42^{\prime} 30^{\prime \prime} \mathrm{W}\right.$, Grid Ref SP 198557) 


\section{Birm-564. Heath End Pumping Sta} $\left(52^{\circ} 14^{\prime} 40^{\prime \prime} \mathrm{N}, 01^{\circ} 39^{\prime} 30^{\prime \prime} \mathrm{W}\right.$, Grid Ref SP 233608)

\section{Birm-565. Budbrooke Pumping Sta
$\left(52^{\circ} 16^{\prime} 45^{\prime \prime} \mathrm{N}, 01^{\circ} 37^{\prime} 35^{\prime \prime} \mathrm{W}\right.$, Grid Ref SP 254648)}

Birm-566. Shrewley Pumping Sta (52 $18^{\prime} 15^{\prime \prime} \mathrm{N}, 01^{\circ} 40^{\prime} 20^{\prime \prime} \mathrm{W}$, Grid Ref SP 222676)

Birm-567. Rowington Pumping Sta (52 $18^{\prime} 45^{\prime \prime} \mathrm{N}, 01^{\circ} 41^{\prime} 30^{\prime \prime} \mathrm{W}$, Grid Ref SP 209687)

Birm-568. Lillington Pumping Sta $\left(52^{\circ} 18^{\prime} 15^{\prime \prime} \mathrm{N}, 01^{\circ} 31^{\prime} 20^{\prime \prime} \mathrm{W}\right.$, Grid Ref SP 326673)

\section{Burton-on-Trent series}

Samples from Triassic Sandstone aquifer at Burton-on-Trent brewery (52 $48^{\prime} 30^{\prime \prime} \mathrm{N}, 01^{\circ} 38^{\prime} 00^{\prime \prime} \mathrm{W}$, Grid Ref SK246232). Coll Dec 1973 and subm by JWL.

\section{Birm-483.}

\section{Birm-484.}

\section{Bath, Somerset}

Sample from Melting Spring in treatment baths near Beau St, Bath, Somerset ( $51^{\circ} 22^{\prime} 50^{\prime \prime} \mathrm{N}, 02^{\circ} 21^{\prime} 45^{\prime \prime} \mathrm{W}$, Grid Ref ST 749647). Coll July 1974 and subm by Isla McTaggart, Dept Geol, Univ Birmingham.

\section{Birm-557.}

$$
-0.65 \quad 20.9 \pm 0.9
$$

General Comment: samples are part of continuing study of ground water dating. Without attempting interpretation of these figures, it is clear that most pumping stations cited above lack appreciable modern recharge.

\section{REFERENCES}

Alexander, M and Worsley, P, 1973, Stratigraphy of a neoglacial end moraine in Norway: Boreas, v 2, no. 3, p 117-142.

Barfield, L H and Fasani, L, 1972, Bemerkungen zum späten Neolithikum und zum Beginn der Bronzezeit in Norditalien: Musaica [Bratislava], v 23, p $45-63$.

Bisat, W S, 1952, Post-glacial peat and Scrobicularia clay near Easington, Yorkshire: Leeds Geol Assoc Trans, v 6, pt 4, p 210-214.

Bishop, W H, 1914, An Oronsay shell mound-a Scottish pre-Neolithic site: Antiquaries Soc Scotland Proc, v 48, p 52-108.

Davidson, Ian, 1974, Radiocarbon dates for the Spanish Solutrean: Antiquity, v 48, p 63-65.

Engel, Frederic, 1966, Le complexe précéramique d'El Paraiso, Pérou: Soc Americanistes Jour [Paris], v 55, p 43-96. 
Gelling, P S, 1959, Excavations at Caynham Camp, near Ludlow; First Interim Report: Shropshire Archaeol Soc Trans, v 56, p 145-148.

Shro 1960, Excavations at Caynham Camp, near Ludlow; Second Interim Report: Shropshire Archacol Soc Trans, v 56, p 218-227. shire Archaeol Soc Trans, v 57, p 91-100.

International Atomic Energy Agency, 1968, Guidebook on nuclear techniques in hydrology: Tech rept ser no. 91, Vienna.

Kidson, C, Haynes, J R, and Heyworth, A, 1974, The Burtle Beds of Somerset_Glacial or Marine? Nature, v 251, no 5472, p 211-213.

Longin, Robert, 1971, New method of collagen extraction for radiocarbon dating: Nature, v 230, p 241-242.

MacAdam, Robert, 1856, Ancient water-mills: Ulster Archaeol Jour, v 4, p 6-15.

Pericot García, D L, 1942, La Cueva del Parpalló: Madrid.

Smith, B N and Epstein, S, 1971, Two categories of ${ }^{13} \mathrm{C} /{ }^{12} \mathrm{C}$ ratios for higher plants: Plant Physiol, v 47, p 380-384.

Stewart, Balfour W, 1914, Notes on a further excavation of ancient dwellings at Skara, in the parish of Sandwick, Orkney: Antiquaries Soc Scotland Proc, v 12, p 344-355.

Tomlinson, M E, 1925, River-terraces of the Lower Valley of the Warwickshire Avon: Quart Jour Geol Soc [London], v 81, p 137-163.

Watson, Edward, 1971, Remains of pingos in Wales and in the Isle of Man: Geol Jour, v 7, p 381-392.

Watson, $\mathbf{E}$ and Watson, S, 1972, Investigations of some pingo basins near Aberystwyth, Wales: 24th internatl geol cong, Montreal, sec 12, p 221-223.

Wimbolt, S E, 1930, Excavations at Holmbury Camp, Surrey: Surrey Archaeol colln, v 38, p 156-170. 